\title{
5,7-Dihydroxytryptamine Lesions of Crayfish Serotonin-Containing Neurons: Effect on the Lateral Giant Escape Reaction
}

\author{
David L. Glanzman ${ }^{\star} \dagger$ and Franklin B. Krasne* \\ *Department of Psychology and Brain Research Institute, University of California, Los Angeles, California 90024, \\ and †Marine Biological Laboratory, Woods Hole, Massachusetts 02543
}

The crayfish's lateral giant escape response, a relatively simple behavioral reaction, is readily modulated in certain situations. For example, when a crayfish is restrained, its lateral giant (LG) fibers-command neurons that mediate the escape responseare strongly inhibited (Krasne and Wine, 1975). Previous work (Glanzman and Krasne, 1983) had suggested that serotonin (5HT) might mediate this restraint-induced inhibition of the escape response. To test this possibility, we attempted to lesion serotonergic neurons in crayfish with the 5-HT neurotoxin, 5,7dihydroxytryptamine (5,7-DHT).

We compared the levels of 5-HT-immunoreactive staining in nerve cords from 5,7-DHT-treated and from normal crayfish to assess 5,7-DHT's effectiveness. Levels of immunoreactive staining, as judged by ratings of the visibility of immunofluorescence, were significantly lower in nerve cords from crayfish that had received injections of 5,7-DHT (1.0-4.0 mg) than in nerve cords from normal crayfish. In addition, some serotonergic neurons in the neurotoxin-treated crayfish developed an abnormal brown pigmentation.

To assess the behavioral consequence of central serotonergic lesions, we compared the responsiveness of escape in crayfish treated with 5,7-DHT (2.0-2.75 mg) and in normal crayfish. The threshold for firing the LGs was significantly lower in restrained neurotoxin-treated animals than in restrained normal animals. Furthermore, the responsiveness of the LGs in neurotoxin-treated crayfish approximated that in crayfish whose nerve cords had been severed between the thorax and abdomen, a procedure known to abolish restraint-induced inhibition (Krasne and Wine, 1975). Finally, 5,7-DHT did not lower the firing threshold of the LGs in unrestrained crayfish; this result rules out a general increase in the excitability of the LGs as an explanation for 5,7-DHT's disruption of restraint-induced inhibition.

Our results imply that the modulation of the crayfish's escape reaction induced by restraint is mediated, at least in part, by central 5-HT.

Monoamines have various physiological actions within the nervous systems of both invertebrates and vertebrates (e.g., Beltz

\footnotetext{
Received July 17, 1985; revised Nov. 12, 1985; accepted Dec. 5, 1985.

This work was supported by United States Public Health Service Grant NS 08108 and by funds from the Grass Foundation. We are most grateful to Dr. Gordon A. Wyse for introducing us to the immunohistochemical techniques employed in this study, to Dr. Barbara S. Beltz for advice on the 5,7-DHT injections, to Zeiss for supplying the microscopic facilities, and to Phil Presley of Zeiss for expert technical assistance. We also thank Terri Teshiba and Drs. Joan Gunther and Sun-Hee Lee for their assistance with some of the experiments, Marvin Nalick for help in preparing the figures, and Harriet Ayers for typing the manuscript.

Correspondence should be sent to Dr. David L. Glanzman at his present address: Howard Hughes Medical Institute, Center for Neurobiology \& Behavior, Columbia University College of Physicians \& Surgeons, 722 West 168th Street, New York, NY 10032

Copyright (C) 1986 Society for Neuroscience $0270-6474 / 86 / 061560-10 \$ 02.00 / 0$
}

et al., 1984; Breen and Atwood, 1983; Fischer and Florey, 1983; Jahr and Nicoll, 1982; Kravitz et al., 1981; Libet, 1979; Madison and Nicoll, 1982; O'Shea and Evans, 1979; Paupardin-Tritsch et al., 1981; Watanabe and Koketsu, 1973). However, the behavioral significance of most of these actions remains obscure. In particular, there are few instances where the physiological actions of an endogenous monoamine have been definitely shown to play a role in the modulation of an animal's behavior. The most compelling evidence for modulatory behavioral roles for monoaminergic physiological actions comes from work on invertebrates. For example, serotonin (5-HT) appears to mediate the behavioral arousal accompanying feeding in Aplysia. Firing of an identified serotonergic neuron-the metacerebral cell (MCC)-enhances several aspects of Aplysia's feeding behavior. Moreover, destroying the MCCs or generally depleting the animal of 5-HT alters various parameters of $A$ plysia's feeding (such as the interval between successive bites) but does not eliminate feeding per se (Kupfermann and Weiss, 1981; Rosen et al., 1983). Release of endogenous 5-HT might also play a modulatory role in the feeding behavior of the medicinal leech (Lent and Dickinson, 1984b). The nature of 5-HT's role in the leech's feeding is less clear than its role in Aplysia's feeding, however, because although 5-HT in the leech is associated with some modulatorytype behavioral effects, depleting this animal of 5-HT abolishes its feeding altogether. This may indicate that 5-HT plays a command rather than a modulatory role in the leech's feeding. Possibly it plays both roles. Serotonin may also play a dual role in another of the leech's behaviors - swimming. Willard (1981) has shown that elevated levels of 5-HT in the blood of leeches increase the probability of swimming by these animals. A likely source for 5-HT in leech blood is the Retzius cells-large serotonergic cells within the central ganglia of leeches. Interestingly, the Retzius cells do not synaptically drive any of the known swim-initiating neurons. Instead, Retzius cells appear to mediate a behavioral state in which the likelihood of swimming is increased. (Lent and Dickinson's work suggests that one such behavioral state may be hunger.) However, there are 5-HTcontaining neurons in leech ganglia whose stimulation can directly (i.e., synaptically) initiate swimming and that appear to be critical to the swimming motor program (Kristan and Nusbaum, 1982/1983). Thus, in the leech, 5-HT seems to participate in both behavioral modulation and behavioral activation.

Another invertebrate whose behavior might be modulated through release of endogenous monoamines is the crayfish. The crayfish is an attractive animal in which to study the potential modulatory behavioral roles of endogenous monoamines. First, it possesses a simple behavioral response, the lateral giant (LG) escape response, which has been well described neurophysiologically (Wine and Krasne, 1982). Second, the threshold for eliciting the response can be modulated either up or down under certain circumstances. For example, when the animal receives a strong, painful stimulus (such as an electrical shock delivered 
to its head or tail), the escape response is facilitated, i.e., sensitized (Krasne and Glanzman, in press). On the other hand, when the crayfish is restrained (for example, by being picked up and held), the escape response is inhibited (Krasne and Wine, 1975). The escape response is also inhibited when the crayfish is feeding (Krasne, 1983). That monoamines might be involved in the modulation of the crayfish's escape was suggested by experiments of Glanzman and Krasne (1983) in which octopamine and 5-HT were applied directly to the crayfish's CNS. Applied octopamine mimicked the facilitatory effects of traumatic stimuli on the escape reaction and applied 5-HT mimicked the inhibitory effects of restraint and feeding on the reaction. It therefore seemed reasonable to suppose that these two monoamines might play modulatory roles in the crayfish's escape behavior, particularly since both $5-\mathrm{HT}$ and octopamine have been shown through high-performance liquid chromatography to be present in significant amounts in the crayfish's CNS (Elofsson et al., 1982). Moreover, 5-HT-like immunoreactive neurons have been identified in the crayfish's brain (Elofsson, 1983).

The purpose of the experiments reported here was to test whether 5-HT mediates restraint-induced inhibition of the crayfish's LG escape response. We injected crayfish with 5,7-dihydroxytryptamine $(5,7-\mathrm{DHT})$, a cytotoxic analog of $5-\mathrm{HT}$, in order to lesion 5-HT-containing neurons; we then looked to see whether the neurotoxin interfered with restraint-induced inhibition. Previous studies have shown that 5,7-DHT selectively lesions serotonergic neurons in lobsters, leeches, and flies (Glover and Kramer, 1982; Lent and Dickinson, 1984b; Livingstone et al., 1981; Nässel and Elekes, 1985). To ascertain the effectiveness of the neurotoxin injections, we compared levels of 5-HT-like immunoreactivity in the CNS of both normal and 5,7-DHT-treated crayfish.

A preliminary account of these results has been published (Gunther et al., 1984).

\section{Materials and Methods}

Procambarus clarkii, weighing 4.5-9.0 gm, were used. They were housed in plastic dishes containing dechlorinated tap water.

\section{5,7-DHT injections}

Animals received injections of $1.0-4.0 \mathrm{mg}$ (total dosage) of 5,7-DHT (Sigma). Some animals received a single injection of the neurotoxin, whereas others received two injections spaced 3-5 d apart. We found no difference in the results from either anatomical or behavioral assessments between giving a certain dose of 5,7-DHT to an animal in one or in two injections; we have therefore combined the results from the two procedures. (In instances where two injections were given, all behavioral assessments were made following the second injection.) The 5,7-DHT was dissolved in normal crayfish saline (Van Harreveld, 1936) at a concentration of $2.5 \times 10^{-2} \mathrm{M}$. The saline was buffered with HEPES (usually 20 InM); its isotonicity was maintained by reducing the $\mathrm{Na}^{+}$ concentration and its $\mathrm{pH}$ was adjusted to 7.2. Sodium L-ascorbate (ICN) was added to the saline at a concentration of either 0.1 or $0.5 \%$ to retard oxidation of the 5,7-DHT. The neurotoxin was injected into the animal's hemolymph with a syringe by two methods. In the first (usual) method, the crayfish was restrained, ventral side up, with gauze cloth and rubber bands in a preparation dish containing cold $\left(4^{\circ} \mathrm{C}\right)$ normal crayfish saline. The syringe needle (Hamilton, 30 gauge) was inserted through the soft cuticle under the first abdominal sternum, and the neurotoxin then injected into the ventral hemolymph sinus. Following the injection the animal's abdomen was allowed to curl up out of the bath to facilitate clotting of the injection wound. After $7 \mathrm{~min}$ the animal was removed from the preparation dish and returned to a holding dish containing fresh water. In the second method, the 5,7-DHT was injected into the hemolymph surrounding the crayfish's brain. Again, the animal was completely immersed in cold normal saline. A small hole was drilled in the dorsal carapace just medial and caudal to one of eyestalks. When hemolymph could be seen flowing from the hole, the neurotoxin was injected into the hole via a syringe with a 23 gauge blunt-tipped needle. Following the injection, the animal was kept in the saline for $7 \mathrm{~min}$ and then placed in a holding dish with fresh water. The two methods of injection yielded similar results.

\section{Nerve cord operations}

Some crayfish had their ventral nerve cords severed between either the fifth thoracic ganglion and the first abdominal ganglion, or between the first and second abdominal ganglia. For this operation, the animal was again immersed in cold saline. A small window was made in the ventral cuticle, and the nerve cord completely severed with a pair of fine dissecting scissors, care being taken not to damage the ventral artery. Recovery procedures were similar to those followed after ventral injections of 5,7-DHT.

\section{Neuroanatomy}

To assess the effects of the 5,7-DHT injections on serotonergic neurons, the CNS of both normal and 5,7-DHT-treated crayfish were processed for 5-HT immunohistochemistry. We closely followed the procedure developed by Beltz and Kravitz (1983) for 5-HT immunohistochemical preparation of lobster CNS tissue with generally excellent results. In brief, dissected crayfish CNS tissues were fixed for 6-12 hr in $0.1 \mathrm{M}$ phosphate buffer ( $\mathrm{pH} 7.2$ ) containing $4 \%$ paraformaldehyde. After fixation, tissues were rinsed for $5-8 \mathrm{hr}$ in $0.1 \mathrm{M}$ phosphate buffer containing $0.3 \%$ Triton $\mathrm{X}-100$ and $0.1 \%$ sodium azide. The tissues were then incubated at $4^{\circ} \mathrm{C}$ for $15-20 \mathrm{hr}$ in antiserotonin antibody (Immunonuclear Corp.) diluted with phosphate/Triton X-100/azide. Following incubation in the anti-5-HT antibody, the tissue was rinsed again with phosphate/Triton X-100/azide for $5-8 \mathrm{hr}$ and then incubated at $4^{\circ} \mathrm{C}$ for $15-20 \mathrm{hr}$ in goat anti-rabbit IgG labeled with fluorescein isothiocynate (FITC) (Boehringer Mannheim Biochemicals). The excess FITC-labeled antibody was rinsed from the tissues with phosphate/Triton X-100/ azide $(5-8 \mathrm{hr}$ ), and then the tissues were rinsed once more in $4 \mathrm{~mm}$ sodium carbonate buffer ( $\mathrm{pH} 9.5)$. The tissues were whole-mounted for viewing in $80 \%$ glycerol in $20 \mathrm{~mm}$ carbonate buffer. Typically, the entire ventral nerve cord, including the subesophageal, thoracic, and abdominal ganglia was processed. The whole-mounted material was viewed with a Zeiss IM 35 epifluorescence microscope. Standard exciter-barrier filter and reflector combination cubes for detecting FITC-labeled antibody were used.

The nervous systems of unprepared, freshly dissected nerve cords of crayfish were also examined for visible effects of 5,7-DHT. Here, the nerve cord was simply pinned out in a Sylgard-lined dish and viewed with a Zeiss stereoscope.

Anatomical preparations were photographed with cameras attached to the microscopes on ASA 400 Ektachrome film.

\section{Tests of $L G$ excitability}

Animals were tested to determine the excitability of their escape responses. Taps of various strengths were delivered to an animal's abdomen, and the strength of the tap necessary to elicit firing of the LGs determined. The activity of the LGs was monitored with extracellular "skewer"-type recording electrodes. The clectrodes wcre a pair of 00 stainless steel insect pins insulated with Epoxylite except for a $0.5 \mathrm{~mm}$ section in the center of the pins. During testing the animal was swaddled with wet gauze and tied down with rubber bands, ventral side up, to a platform made of modeling clay and Plexiglas rods (Fig. 5A). The platform was fixed to the bottom of a preparation dish filled with dechlorinated water. The skewer electrodes were inserted into the animal's abdomen through the soft ventral cuticle and positioned so that the uninsulated section of the electrodes rested on the dorsal surface of the nerve cord. Care was taken to damage neither the nerve cord nor the ventral artery that runs beneath it. The electrodes were placed on the connectives between the third and fourth, and between the fourth and fifth, abdominal ganglia of the nerve cord (Fig. $5 B$ ). Stimuli were delivered by a tapper consisting of a 14-cm-long Plexiglas rod, $6 \mathrm{~mm}$ in diameter, which was affixed to the center of a standard 5 in., $8 \Omega$ Radio Shack speaker. The speaker was driven by a Grass AM 8 audio monitor; the strength of the stimuli could be varied by varying the output of the AM 8. Pulses from a Grass SD 9 stimulator drove the AM 8 and triggered a sweep from a storage oscilloscope that monitored the electrical activity of the nerve cord. The tip of the tapper, filed down to $3 \mathrm{~mm}$ with a pencil sharpener, was positioned approximately $1 \mathrm{~mm}$ from the cray- 
Figure 1. Photograph of a crayfish minutes after receiving an injection of $1.5 \mathrm{mg}$ of 5,7-dihydroxytryptamine (5,7-DHT). The drug was injected directly into the animal's ventral hemolymph sinus. It produced a pronounced postural flexion in the animal.

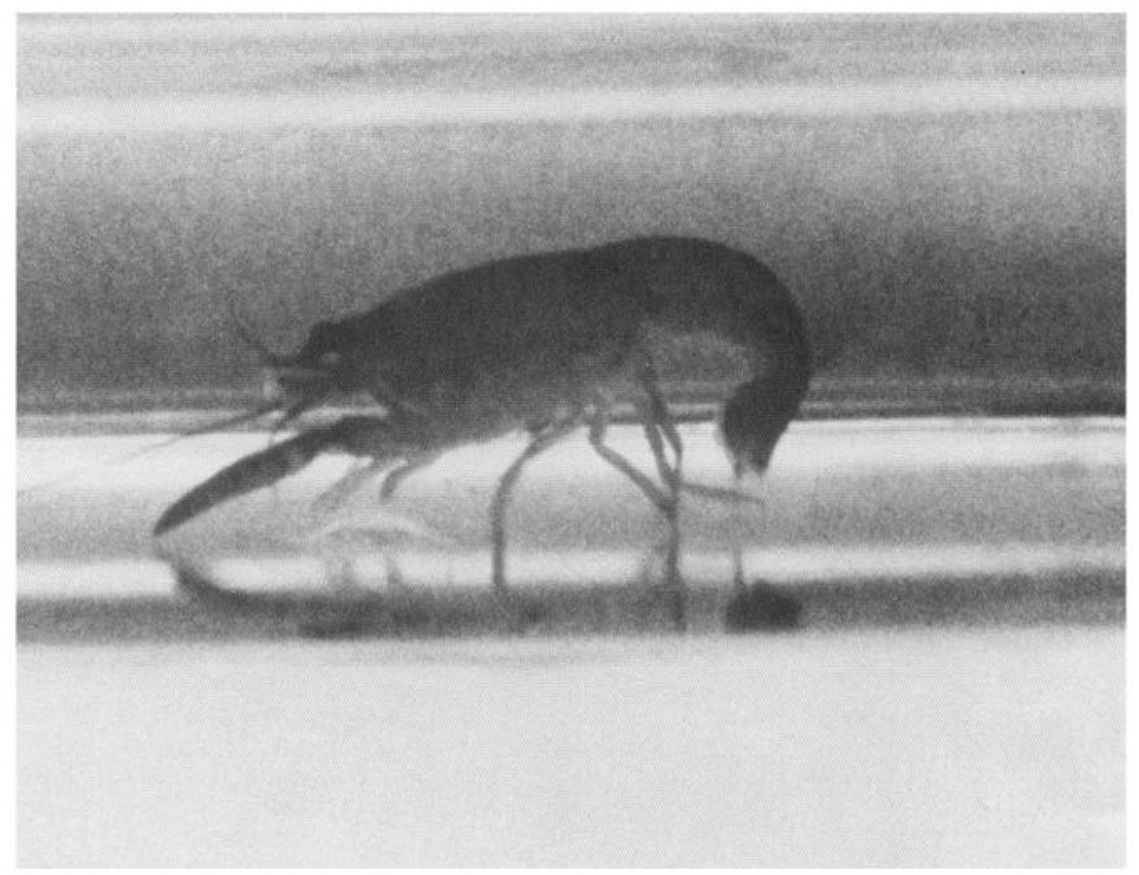

fish's abdomen; taps were delivered to one side of the fifth pleural plate. The tapper was repositioned between trials if necessary so that the tip remained at a constant distance from the pleural plate. The strengths of the taps were calibrated with a Grass FT03C force transducer; they ranged from 0 to $444 \mathrm{gm}$. A semischematic diagram of the stimulating arrangement is shown in Figure $5 A$.

In some experiments, described under Results, the threshold for firing the LGs was determined for unrestrained, freely moving crayfish. Here, stimulation and recording were by means of chronically implanted electrodes. The recording electrodes were like those used to test restrained animals and were positioned similarly. The stimulating electrodes, also skewer-type electrodes, were placed on either the dorsal or ventral surfaces of roots two, three, and four of the sixth abdominal ganglion. Test stimuli were $0.1 \mathrm{msec}$ negative pulses of varying voltages, which were isolated from ground by a transformer.

The occurrence of an LG escape reaction was recognized by LG spikes followed by large muscle potentials. Since firing of the LGs was usually recorded at the electrodes within $10 \mathrm{msec}$ of the stimulus, we used a 20 msec oscilloscope sweep; any LG firing that occurred later than $20 \mathrm{msec}$ was not counted as a response.

\section{Results}

\section{Immediate behavioral effect of 5,7-DHT injections}

Within minutes after an injection of 5,7-DHT (1.0-4.0 mg), crayfish invariably exhibited sustained, tonic flexion of their abdomens (Fig. 1). This postural flexion typically lasted for several hours following an injection of the toxin. Animals receiving control injections of the carrier vehicle (crayfish saline and sodium L-ascorbate) by itself never exhibited sustained postural flexion. Interestingly, this behavioral effect of 5,7-DHT appears indistinguishable from that caused by injecting lobsters and crayfish with large quantities of 5-HT (Livingstone et al., 1980; D. Glanzman, unpublished observations). To our knowledge, this is the first reported instance of 5,7-DHT having a short-term behavioral effect that mimics that of applied 5-HT. Our result suggests that 5,7-DHT, in the concentrations reached in these injections, may act as a 5-HT agonist or that it may stimulate release of 5-HT from the terminals of serotonergic neurons, or both.

\section{5,7-DHT injections reduce 5-HT-like immunoreactive staining of the ventral nerve cord}

Ventral nerve cords from eight normal, uninjected crayfish and from five crayfish that had received injections of 5,7-DHT were processed for 5-HT immunohistochemistry. The dosages of 5,7DHT received by the five neurotoxin-treated animals were: 4.0 , $3.0,3.0,2.75$, and $1.0 \mathrm{mg}$. We waited 2-6 d after injections before processing the tissue from the neurotoxin-treated animals.

The pattern of immunoreactive staining seen in the ventral nerve cords from the normal (control) animals is generally like that reported for lobsters by Beltz and Kravitz (1983). Thus, there is a pair of immunoreactive cell bodies in each of the five thoracic ganglia (Fig. $2 B$ ); there are also three large (approximately $100 \mu \mathrm{m}$ ) immunoreactive cell bodies in the first abdominal ganglion consisting of a lateral pair of cells and a medial, unpaired cell (Fig. 2C). Another feature of the normal crayfish ventral nerve cord is bundles of longitudinally running immunoreactive fibers. Three pairs of fiber bundles that are particularly prominent run from the subesophageal ganglion through at least the first abdominal ganglion. These were also seen by Beltz and Kravitz (1983) in the lobster nerve cord and were labeled by them the midline, central, and lateral fiber bundles (Fig. 2, $A, B$ ). Other points of similarity between the crayfish and lobster nerve cords include the presence of lateral regions of immunoreactive neuropil within the thoracic and abdominal ganglia, as well as dense plexes of varicosities surrounding second nerve roots of thoracic ganglia. These immunoreactive varicosities may represent sites for peripheral release of 5-HT (Livingstone et al., 1981). One difference was apparent between the patterns of immunoreactive staining in the lobster and crayfish nerve cords: Whereas Beltz and Kravitz found immunoreactive cell bodies in every abdominal ganglion, we only rarely found immunoreactive somata behind the first abdominal ganglion, and never in the sixth (last) ganglion. However, because the patterns of 5-HT immunoreactivity in nerve cords of lobsters and crayfish are quite similar rostral to the second abdominal ganglion, it may be that 5-HT-containing cell bodies are actually 

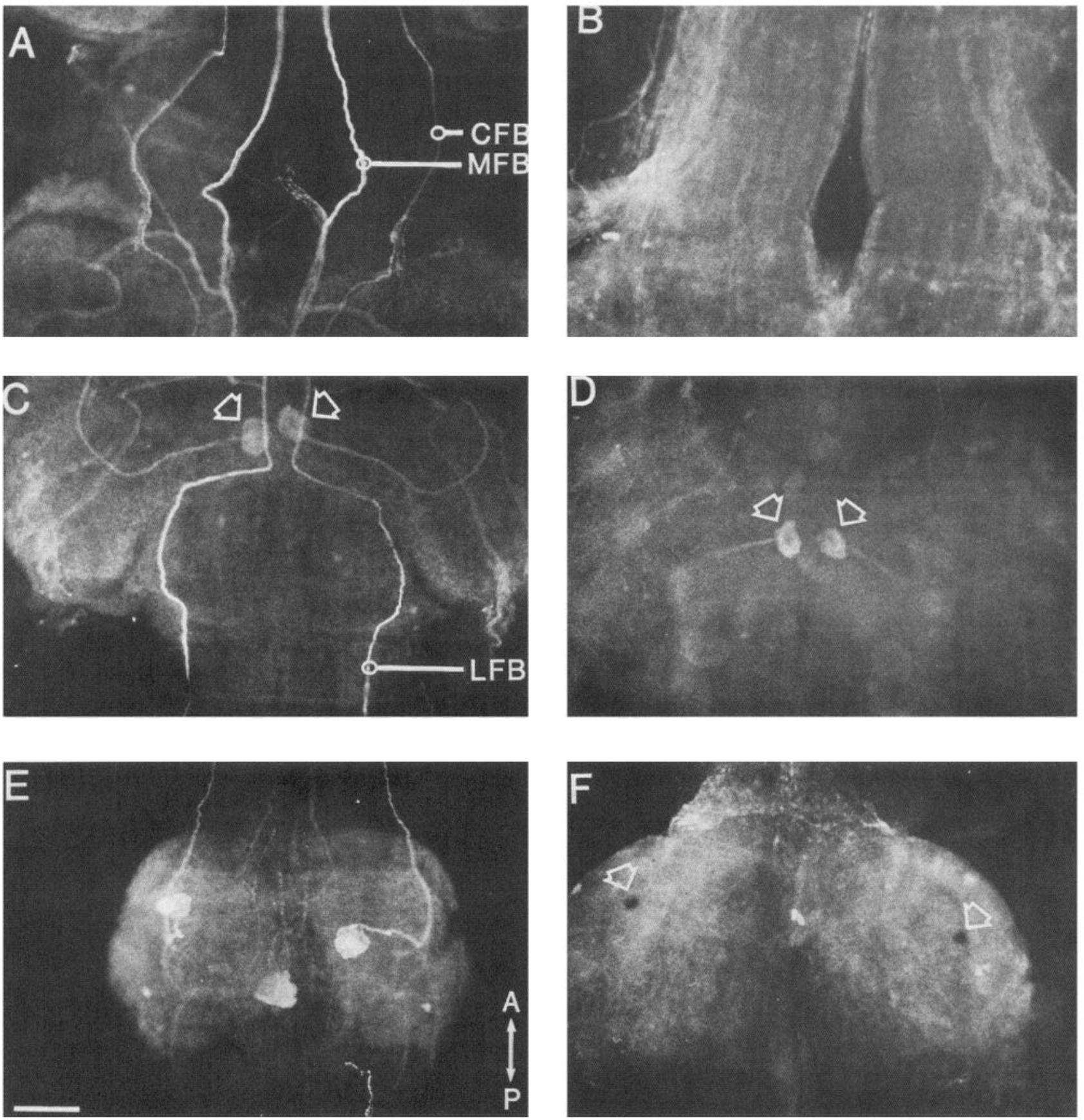

Figure 2. Photographs of whole-mounted central tissue from normal and 5,7-DHT-treated crayfish processed for serotonin (5-HT)-like immunoreactivity. The normal tissue is shown at left; the tissue from the neurotoxin-treated animals is shown at right. Calibration bar, $200 \mu \mathrm{m}$. $A$, Connectives between the third and fourth thoracic ganglia from a normal animal. Note the rich system of immunoreactive fibers. In particular, there are two prominent pairs of fiber bundles shown that run longitudinally in the ventral nerve cord, the midline fiber bundles (MFBs) and the central fiber bundles $(C F B s)$. A third pair of fiber bundles, the lateral fiber bundles $(L F B S)$, is not readily apparent in this photograph (but see $C$ and $E$ ). $B$, Connectives between the third and fourth thoracic ganglia from an animal treated with 4.0 mg of 5,7-DHT. No immunoreactive fiber bundles are visible. $C$, Fifth thoracic ganglion from a normal animal. The photograph is centered on the posterior part of the ganglion; it shows the LFBs, which are quite prominent in the connectives between the fifth thoracic and first abdominal ganglia. Note that the LFBs arriving from the abdomen turn medially in the fifth thoracic ganglion, where they become part of the MFBs. This ganglion contains a medial pair of large immunoreactive cells (arrows) located approximately in the center of the ganglion. Each of these cells can be seen to give rise to a large immunoreactive process. Each process turns anteriorly to join the ipsilateral LFB in the anterior segment of the ganglion (not shown). $D$, Fifth thoracic ganglion from an animal treated with $1.0 \mathrm{mg}$ of 5,7-DHT. The photograph is centered on the medial pair of immunoreactive cells (arrows). Neither the LFBs nor the MFBs can be seen with the ganglion. Also, the immunoreactive processes arising from the cells are only faintly and incompletely visible. E, First abdominal ganglion from a normal animal. There is a lateral pair of large immunoreactive cells and a large, unpaired medial cell. Each of the lateral cells gives rise to an immunoreactive process that enters the ipsilateral connective, joining the first abdominal and fifth thoracic ganglia; there, the process becomes part of one of the LFBs shown in $C$. F. Fifth thoracic ganglion from an animal treated with 4.0 mg of 5,7-DHT (the same animal contributing the tissue shown in $B$ ). Neither immunoreactive cell bodies nor their processes are visible. In the positions usually occupied by the lateral cell bodies are brown spots (arrows). A brown spot corresponding to the medial cell body is not visible in the photograph but was seen when this ganglion was inspected just after it had been dissected out of the animal (see Fig. $4 B$ ). 
Table 1. Structures rated for visibility of 5-HT immunofluorescent staining in tissue from normal and 5,7-DHT-injected crayfish

Medial fiber bundles ${ }^{a}$ in the connectives between the $3 \mathrm{rd}$ and 4 th thoracic ganglia

Central fiber bundles in the connectives between the $3 \mathrm{rd}$ and 4 th thoracic ganglia

Medial fiber bundles in the connectives between the 4th and 5th thoracic ganglia

Anteromedially projecting fibers from the cell bodies in the 4th thoracic ganglion

Lateral fiber bundles in the connectives between the 5 th thoracic and 1st abdominal ganglia

Cell bodies, 3rd thoracic ganglion

Cell bodies, 4th thoracic ganglion

Cell bodies, 5 th thoracic ganglion

Lateral pair of cell bodies, 1st abdominal ganglion

Unpaired medial cell body, lst abdominal ganglion ${ }^{b}$

All structures, except where noted, are presented bilaterally in the crayfish's nerve cord. Thus, for each bilateral structure, two independent ratings were made.

a Names of the structures are after Beltz and Kravitz (1983).

${ }^{b}$ Only one such structure is present in the nerve cord.

present in the caudal regions of the crayfish's nerve cord, but that there is some barrier to the antibody in these regions. Had we used sectioned material or nerve cords from very young animals, we might have found a pattern of 5-HT immunoreactivity in the abdominal nerve cord resembling that seen in the lobster.

The immunoreactive staining of tissue from 5,7-DHT-treated animals was conspicuously fainter than that of tissue from normal animals. Fibers and other processes appeared more susceptible to loss of fluorescence due to 5,7-DHT treatment than did cell bodies (compare Fig. 2, $A$ to $D$ and $B$ to $E$ ). However, in some cases the fluorescence of even large cell bodies was completely eliminated (compare Fig. 2, $E$ and $F$ ).

To quantify the apparent difference between the levels of immunoreactive staining in the tissues from the untreated and 5,7-DHT-treated animals, we rated the visibility of the immunofluorescence in these tissues. Nineteen target structures (Tablc 1) werc sclceted for rating. (The tissue contributed by each animal did not necessarily contain all 19 target structures, although the majority did. A minimum of five target structures was rated for each animal's tissue.) The ratings were carried out on whole-mounted tissue viewed with an epifluorescence microscope. Each target structure was given a subjective visibility rating of between 0 and +2 , with 0 indicating a complete absence of immunofluorescence and +2 indicating a high level of immunofluorescence. The rater was blind as to which animal had contributed the tissue he rated. The scores for all the target structures rated for a given animal were summcd, and a mcan rating score was determined for the animal. The mean rating scores for the normal and 5,7-DHT-treated animals are shown in Figure 3. There was no overlap between the scores for the normal and for the 5,7-DHT-treated groups: All of the normal animals had higher scorcs than did the toxin-treated animals. This difference between the visibility ratings was highly significant $(P<0.002$; two-tailed Mann-Whitney $U$ test).

One way in which the immunofluorescence results might have been biased would have been if relatively different numbers of scrotoncrgic fibcrs had becn used as target tissues in the neurotoxin-treated and control groups. This is because 5,7-DHT had a greater apparent effect on fiber bundles than on cell bodies within the crayfish's nerve cord (see, e.g., Fig. $2 D$ ). If the target tissues for the neurotoxin-treated group consisted largely of fiber

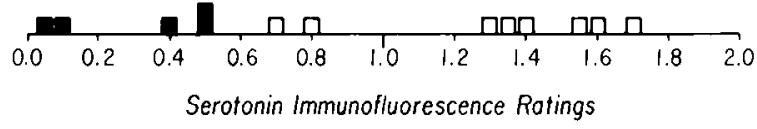

Figure 3. Summary of 5-HT immunofluorescence ratings. Each square represents the mean score for one animal's nerve cord based on ratings made on several different target structures (see Table 1). There was no overlap between the mean scores for the normal and 5,7-DHT-treated animals. We also transformed the raw fluorescence ratings to standard scores to control for the possibility that anatomical targets having high immunofluorescence were more often rated in normal animals. For each target structure rated, an animal was given a transformed score ( $z$ score) according to the formula, $\left(X_{i}-\bar{X}\right) / \mathrm{SD}_{x}$, where $X_{i}$ is the animal's raw rating on a particular target structure, $\bar{X}$ is the mean rating for that structure for all animals, and $\mathrm{SD}_{x}$ is the standard deviation of all the ratings made on that structure. The results from the data analysis using the mean $z$ scores were the same as those from the analysis using the mean raw scores. In both cases, the difference between the scores for normal and those for the 5,7-DHT-treated groups was statistically significant $(p<0.002$; two-tailed Mann-Whitney $U$ test).

bundles, then this could have accounted for our results. To see if such a bias might be present in our data, we counted the total numbers of fibers and cell bodies rated for the two groups of nerve cords. For the five neurotoxin-treated nerve cords, a total of 42 fibers and 36 cell bodies was used as target tissues; for the eight control nerve cords, a total of 64 fibers and 57 cell bodies was used. The ratios of cell bodies to fibers in the target tissues for the two groups were thus practically identical $(0.86$ for the toxin-treated group vs 0.89 for the controls). Therefore, our results cannot be explained by a bias due to unequal numbers of fibers and cell bodies rated for the two groups.

As a second control for possible selection biases in the immunofluorescence ratings, the raw scores were transformed to standard (z) scores (see the legend to Fig. 3). The results from the data analysis using the $z$ scores were similar to those using the raw scores: Again, there was no overlap between the scores for the normal and 5,7-DHT-treated groups, and the difference between the ratings for the two groups was highly significant $(P<0.002$; two-tailed Mann-Whitney $U$ test $)$.

\section{5,7-DHT injections cause 5-HT'-containing cell bodies to develop a brown pigmentation}

Some central ganglia from neurotoxin-treated crayfish contained brown spots; the positions of these spots matched those of yellow-fluorescing cell bodies in corresponding ganglia from normal crayfish (compare Fig. 2, $E$ and $F$ ). Moreover, these brown spots were readily visible in unprocessed, freshly dissected central tissuc (Fig. 4). The brown spots appcar to bc abnormal serotonergic cell bodies. It has been previously reported (Glover and Kramer, 1982; Lent and Dickinson, 1984a, b) that serotonergic cells in central ganglia from the leech also turn brown following treatment with 5,7-DHT. In the crayfish not every apparent scrotonergic cell turned brown following the neurotoxin injections. Some cells (for example those in the fifth thoracic and first abdominal ganglia) were particularly likely to develop brown pigmentation after 5,7-DHT treatment. Also, we found some brown spots in neurotoxin-treated tissue for which we could not find yellow-fluorescing counterparts in normal tissue processed for immunohistochemistry. In particular, we regularly found what appeared to be a cluster of brown spots at the base of sixth abdominal ganglia of 5,7-DHT-treated animals (Fig. 4C), although we never saw immunoreactive cell 

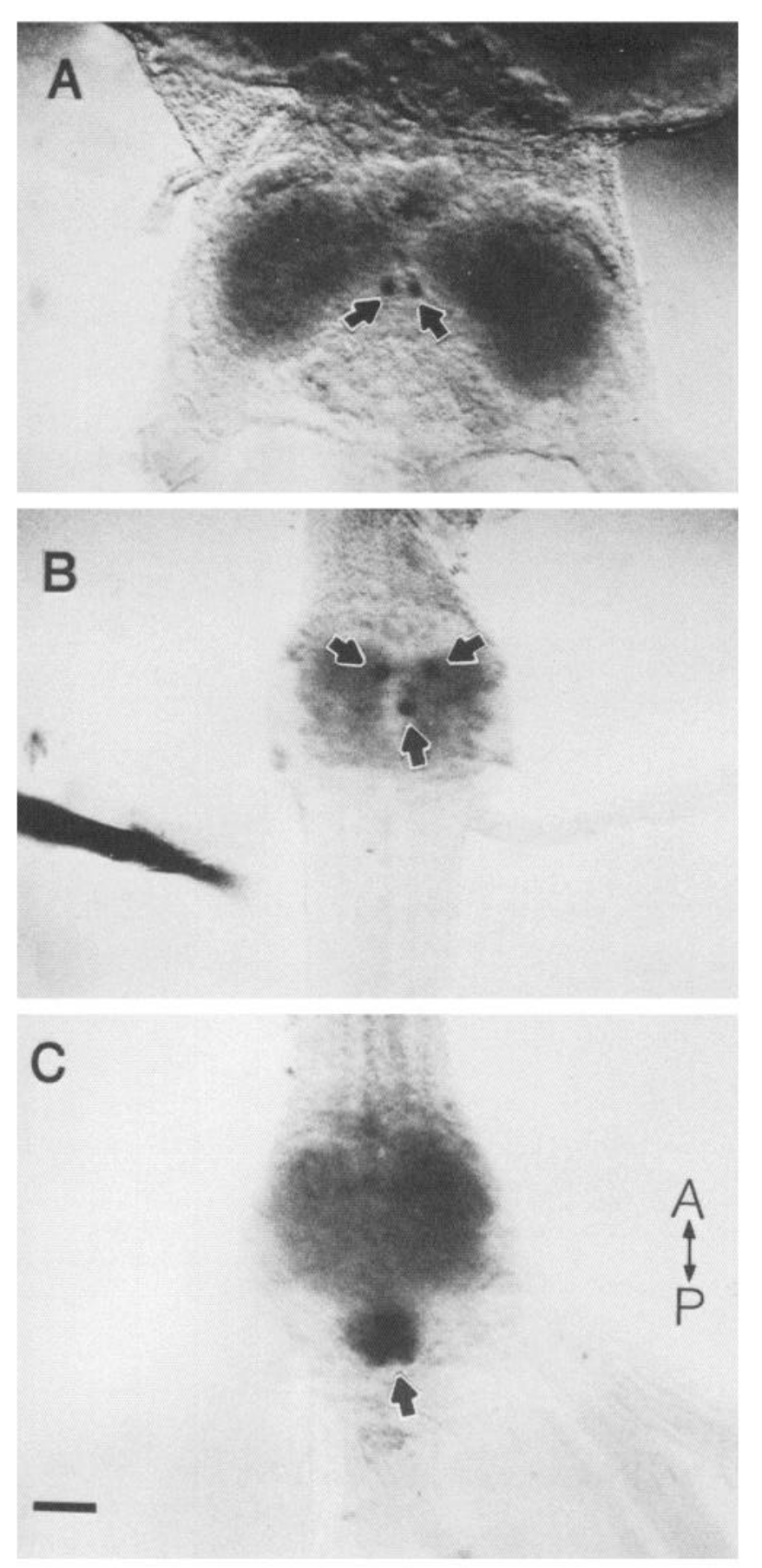

Figure 4. Photographs of freshly dissected, unprocessed ganglia from crayfish treated with 5,7-DHT. The ganglia contain prominent brown spots; these are never seen in ganglia from normal crayfish. Calibration bar, $200 \mu \mathrm{m}$. $A$, Fifth thoracic ganglion from an animal that had received $1.0 \mathrm{mg} \mathrm{5,7-DHT}$. There are two brown spots (arrows) in the ganglion; their positions correspond to those of a pair of 5-HT immunoreactive cells seen in the normal ganglion. Compare with Fig. $2 C$. B, First abdominal ganglion from an animal that had received $2.0 \mathrm{mg} 5,7-\mathrm{DHT}$. There are three brown spots (arrows) in the ganglion; their positions correspond to those of three 5-HT immunoreactive cells seen in the normal ganglion. Compare with Figure $2 E$. (Note that the lateral cell bodies in Fig. $2 E$ are displaced somewhat from their true positions within the ganglion because of whole-mounting.) The black figure at the left of the photograph is a minuten pin. $C$, Sixth abdominal ganglion from an animal that had received $2.5 \mathrm{mg} 5,7-\mathrm{DHT}$. There is a large brown spot at the base of the ganglion (arrow). This spot, as revealed by inspection at higher magnification, is actually a cluster of smaller

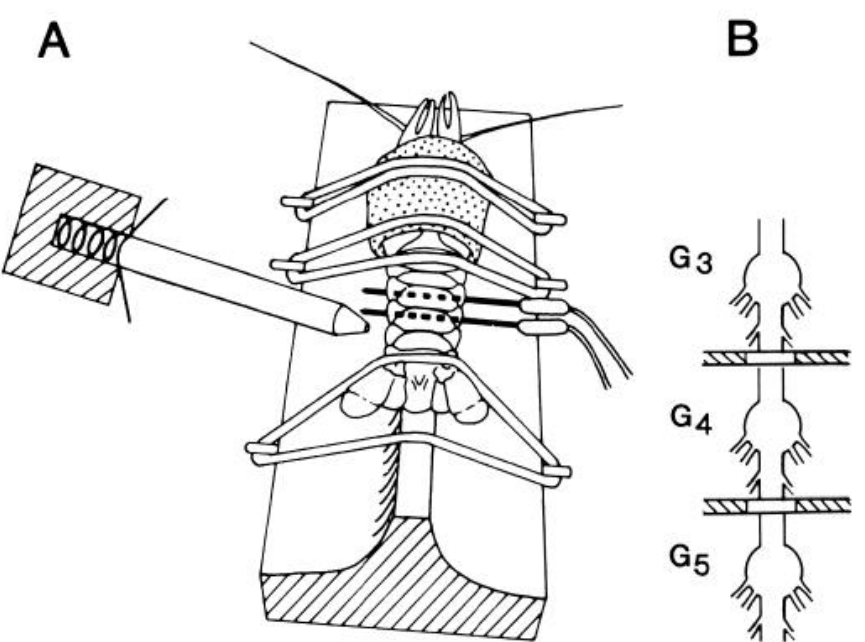

Figure 5. Experimental arrangement for testing restraint-induced inhibition. $A$, Schematic diagram of crayfish during testing. The animal was fastened to a test platform, ventral side up, with rubber bands; its thorax and claws were swaddled with wet gauze cloth. Extracellular recording electrodes were inserted into the animal's abdomen and positioned on the dorsal surface of the ventral nerve cord (see $B$ ). Afferent input to the lateral giants ( $L G s$ ) was stimulated by taps delivered to the abdomen with a tapper. $B$, Arrangement of the recording electrodes. The uninsulated section of each electrode was situated on the dorsal surface of the nerve cord to monitor the extracellular activity of the LGs. The anterior electrode was positioned between the third and fourth abdominal ganglia $\left(G_{3}\right.$ and $\left.G_{4}\right)$; the posterior electrode was positioned between the fourth and fifth abdominal ganglia $\left(G_{4}\right.$ and $\left.G_{5}\right)$.

bodies in sixth abdominal ganglia from normal animals. Possibly, these represent non-serotonergic cells affected by the neurotoxin. However, as judged by the excellent correlation between brown spots and cells stained by 5 -HT immunohistochemistry in the more rostal areas of the crayfish's nerve cord, the neurotoxin appears to be quite selective for serotonergic pathways in crayfish. Also, the lobster's sixth abdominal ganglion contains apparent serotonergic cell bodies, some of whose positions approximate those of the brown spots seen in the toxin-treated sixth abdominal ganglion of crayfish (see Beltz and Kravitz, 1983, Fig. 3). Therefore, the brown spots in the sixth abdominal ganglion might well represent serotonergic cells revealed by the neurotoxin that were not revealed by the 5-HT antibody.

\section{Effect of 5,7-DHT on restraint-induced inhibition of the $L G$ response}

The integrity of restraint-induced inhibition of the LGs was tested in seven animals that received $2.0-2.75 \mathrm{mg}$ of 5,7-DHT. We waited at least $2 \mathrm{~d}$ following the injections before performing the tests. All seven animals survived at least $24 \mathrm{hr}$ after testing. (We excluded the data from tests on three other animals that did not survive for $24 \mathrm{hr}$ after testing.) The effectiveness of restraint-induced inhibition in the seven neurotoxin-treated crayfish was compared to that in eight normal, uninjected crayfish. During the tests, the animals were restrained, ventral side up, and attempts were made to elicit LG escape responses with taps from a Plexiglas rod. Firing of the LGs (and hence elicitation of the escape response) was monitored with extracellular electrodes (see Fig. 5 and Materials and Methods). The protocol for the tests was as follows. After the recording electrodes had been positioned, the animal was given six to eight preliminary

spots. No 5-HT immunoreactive cells were seen in sixth abdominal ganglia from normal animals (see text). 


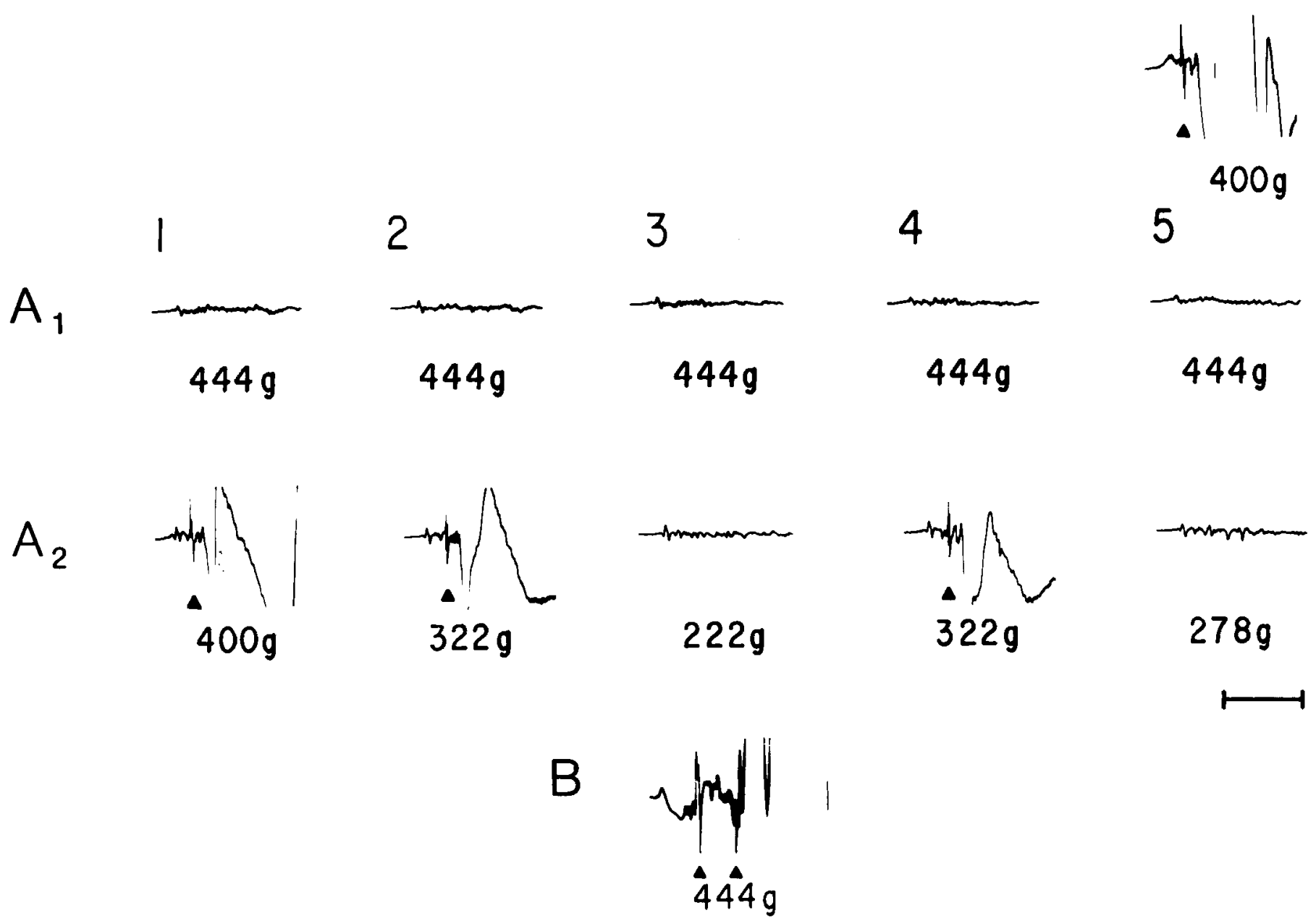

Figure 6. Oscilloscope records from tests of lateral giant $(L G)$ excitability. Each test consisted of five trials. The stimuli's calibrated strengths on the trials are shown below the records. Solid triangles, Firings of the LGs. The large deflections on the records following the LG spikes represent electrical potentials from the phasic flexor muscles that drive escape tailflips. Calibration bar, $10 \mathrm{msec}$. $A_{1}$, Test on a normal animal. The stimuli elicited no LG responses. Inset above record of Trial 5, Preliminary test trial on this animal. An LG response was elicited during the preliminary trial, thereby indicating that the animal's LG system was intact. $A_{2}$, Test on a 5,7-DHT-treated animal. Lateral giant responses were elicited on three of the test trials. $B$, Record from a trial on another 5,7-DHT-treated animal. A single stimulus elicited two LG spikes.

test trials at once per 3 min, each trial consisting of a tap to the animal's abdomen. The preliminary trials were included in order to allow any sensitization (see Krasne and Glanzman, in press) due to insertion of the electrodes time to wane and to acclimate the animal to the testing procedure. During the preliminary trials, the approximate stimulus threshold for eliciting the LG response was tracked. Afterward, the animal was given 5 min of rest and then five test trials, one per $3 \mathrm{~min}$, during which the LGs' threshold was again tracked. The data analyses were based on the results from these latter test trials.

Typical results from the tests are shown in Figure 6. The control (normal) animal (Fig. $6 A_{\text {, }}$ ) completely inhibited its LG firing during the five test trials, although the stimulus was at maximal strength $(444 \mathrm{gm}$ ). The 5,7-DHT-treated animal (Fig. $6 A_{2}$ ), by contrast, responded with LG firing (indicated by the solid triangles) and tail flips on three of the five test trials. These results suggest that restraint-induced inhibition was impaired in the neurotoxin-treated animal. Also, one of the neurotoxintreated animals responded to test stimuli with multiple LG spikes (Fig. 6B). The occurrence of multiple spikes was never seen during tests of normal animals. Multiple LG spikes are somewhat unusual because the LGs typically inhibit their own firing for about $90 \mathrm{msec}$ following a spike through a recurrent inhib- itory pathway (Roberts, 1968). However, multiple LG spikes to single taps have been seen in unrestrained, freely moving crayfish (Wine and Krasne, 1972).

For statistical purposes, each animal was given a single "response proportion" score. The response proportion score represented the proportion of trials during which the animal gave an LG response when the stimulus's strength was between 384 and $444 \mathrm{gm}$. (This interval was chosen because the threshold for most of the animals that responded to the stimuli was within this range.) In a few cases animals that responded to relatively weak stimuli were tested only with stimuli less than $384 \mathrm{gm}$. In such cases it was assumed that an animal would have responded at least as much to stimuli in the 384-444 gm range as to the weaker stimuli. (Extensive experience with this reflex in F.B.K.'s laboratory has shown that response probability never declines as a result of increasing stimulus strength.) In cases where the response proportion for the weaker stimuli was unity, this value was simply entered in the analysis. Cases where the response proportion for the weaker stimuli was less than unity were treated as described below.

Some animals were tested more than once. Their response proportion scores were determined by pooling the results from their individual tests. 


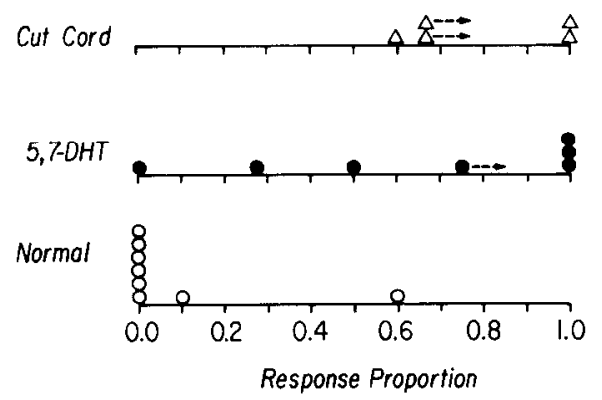

Figure 7. Group data from tests of LG excitability. Each symbol, A "response proportion" score for a single animal based on the proportion of trials during which the animal gave an LG response when the stimulus's strength was between 384 and $444 \mathrm{gm}$ (except as noted otherwise in the text). A score of 1.0 indicates the animal responded on every trial, whereas a score of 0.0 indicates the animal did not respond on any trial. The arrows arising from some of the symbols indicate that the assigned scores may underestimate the animals' true responsiveness (see text). The difference between the scores for the normal and 5,7DHT-treated animals was statistically significant $(P<0.01$; two-tailed $t$ test on the arcsin-transformed proportion data); the difference between the scores for the cut-cord and 5,7-treated animals was not statistically significant $(P>0.5$; two-tailed $t$ test on the arcsin-transformed proportion data).

Figure 7 summarizes the response proportion data. The data point representing the response proportion score of one of the 5,7-DHT-treated animals tested only with stimuli below 384 gm has a rightward-pointing arrow affixed to it; the arrow serves to indicate that this animal's response proportion to 384-444 gm stimuli would probably have been greater (see above). All but one of the 5,7-DHT-treated animals (solid circles) showed some responsiveness to the test stimuli, as indicated by their having scores greater than 0.0 . Only two of the normal animals (open circles), however, responded at all to the stimuli. This difference between the two groups was statistically significant $(P<0.01$; two-tailed $t$ test on the arcsin-transformed proportion data).

Both behavioral and anatomical data were obtained from only one of the 5,7-DHT-treated animals. This raises the possibility that at least some of the animals exhibiting behavioral deficits might not have had central serotonergic lesions. However, we believe this is unlikely. For one thing, an injection of even as little as $1 \mathrm{mg}$ of the neurotoxin produced significant damage to the crayfish's serotonergic pathways (see Fig. 2D). The dosages of 5,7-DHT used in the behavioral studies represent the middle range of those used in the anatomical assessments of the toxin's effect. Since, as Figure 3 shows, there was no overlap between the distributions of ratings of 5-HT immunofluorescence for the normal and 5-7-DHT-treated nerve cords, it is reasonable to conclude that the neurotoxin depleted 5-HT levels in all of the 5,7-DHT-treated animals examined behaviorally.

Comparison of the effects of 5,7-DHT injections with those of cutting the nerve cord

Krasne and Wine (1975) have shown that severing the ventral nerve cord at the thoracic-abdominal junction completely abolishes restraint-induced inhibition of the LG response. In order to provide a standard for determining how effective our 5,7DHT lesions had been, we tested five animals (hereafter called "cut-cord" animals) whose nerve cords had been completely severed, thereby isolating the abdominal nerve cord from the rostral CNS. The animals were allowed to recover from surgery for $3 \mathrm{~d}$ to 2 weeks before testing. The data from the tests on the cut-cord animals (represented by the open triangles) are summarized in Figure 7 . (The meaning of the rightward-pointing arrows is as explained above.) As expected, the probability of

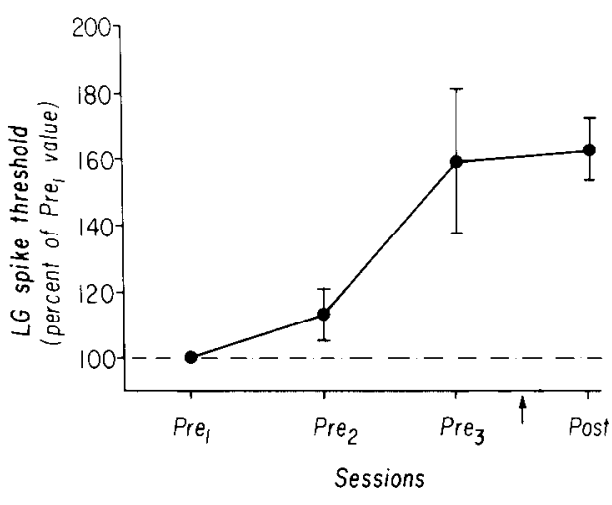

Figure 8. Effect of 5,7-DHT on the LG spike thresholds in unrestrained crayfish. The responsiveness of the LGs was tested in six animals before and after an injection of 5,7-DHT (2.0-2.75 mg). Stimulation and recording were carried out by means of implanted electrodes. The stimulus threshold for eliciting an LG response from each animal was determined during four different test sessions: A session $2 \mathrm{~d}$ before the 5,7-DHT injection $\left(\mathrm{Pre}_{1}\right)$; a session $1 \mathrm{~d}$ before the injection $\left(\mathrm{Pre}_{2}\right)$; a session immediately prior to the injection $\left(\mathrm{Pre}_{3}\right)$; and a session 2-3 d after the injection (Post). During each of the four test sessions, the animals were given 40 stimulus trials; the values shown in the figure represent the stimulus thresholds for trials 11-20 of a given session, where "threshold" was taken to be the value halfway between the minimum stimulus voltage producing an LG response and the maximum voltage not producing one. The mean stimulus thresholds during each session for the six animals are expressed as a percentage of each animal's threshold during the Pre, session. Bars, \pm SEM. The 5,7-DHT injection (arrow) occurred between trials 20 and 21 of the third session.

eliciting an escape response from restrained crayfish increased dramatically when the LG reflex circuitry was isolated from descending central input. It appears that the 5,7-DHT-treated animals were, as a group, somewhat less responsive during restraint than were the cut-cord animals; however, these two groups were certainly more similar to each other than either was to the normal group. A $t$ test comparing the response proportion scores for the cut-cord and 5,7-DHT animals (Fig. 7) failed to attain significance $(P>0.5$; two-tailed $t$ test on the arcsin-transformed proportion data).

Effect of 5,7-DHT on the LG response in unrestrained animals We were concerned that 5,7-DHT might have increased the responsiveness of restrained crayfish not by disrupting restraintinduced inhibition but, rather, by generally increasing the LGs' excitability. Thus, injections of 5,7-DHT might have led to sensitization of the escape reaction (Krasne and Glanzman, in press) or to the removal of a source of entirely tonic inhibition. In order to exclude these possibilities, we tested the excitability of the reaction in freely moving crayfish before and after injections of 5,7-DHT. To achieve stimulus control in unrestrained animals, we used brief shocks via implanted electrodes to stimulate the reaction; we have previously used this method to study sensitization of escape in unrestrained crayfish (Krasne and Glanzman, in press). The shocks were delivered to sensory roots in the sixth abdominal ganglion. The threshold stimulus voltage for firing the LGs was first determined on three successive days prior to 5,7-DHT injections. Immediately after the third determination of the LGs' threshold, six animals were injected with 5,7-DHT. (Different animals were used in the experiments on restrained and unrestrained crayfish, but the animals in the two experiments were given exactly the same range of dosages of the ncurotoxin, 2.0-2.75 mg.) Two to threc days after the injections, the LGs' threshold was determined again. As Figure 8 shows, the threshold for eliciting an LG response from the unrestrained animals during this postinjection test was typically 
equal to, or somewhat above, the response threshold just prior to injection of the neurotoxin.

\section{Discussion}

\section{Selectivity of 5,7-DHT}

The present results, together with those of Glanzman and Krasne (1983), imply that the modulatory effects of restraint on the crayfish's $L G$ reaction are mediated by a descending serotonergic system. However, a problem for this interpretation of the results is that the neurotoxin might have affected not only serotonergic cells, but also neurons containing other transmitters. For example, injections of 5,7-DHT have been reported to damage catecholaminergic pathways in mammals (Baumgarten et al., 1982). Studies on invertebrates, however, have agreed in finding excellent selectivity of 5,7-DHT for serotonergic pathways (Glover and Kramer, 1982; Lent and Dickinson, 1984b; Livingstone et al., 1981; Nässel and Elekes, 1985). Two of these studies involved other arthropods (Livingstone et al., 1981; Nässel and Elekes, 1985), one of which was the lobster. Moreover, these two studies employed electron microscopy to confirm that the neurotoxin's effects were confined to serotonergic terminals. Although we did not examine 5,7-DHT's effect on non-serotonergic transmitters in the crayfish, indirect evidence from our study suggests that the neurotoxin was quite specific in its effects. First, injections of 5,7-DHT always caused crayfish to adopt a posture (characterized by tonic flexion) identical to that produced by $5-\mathrm{HT}$; this indicates that the neurotoxin either selectively activates serotonergic neurons or causes the release of transmitter from their terminals. Second, except for a group of cells in the sixth abdominal ganglion (which may, in fact, prove to be serotonergic), all cells that developed brown pigmentation due to 5,7-DHT treatment appeared to contain 5-HT based on immunohistochemistry. We cannot rule out the possibility, however, that the neurotoxin affected neurons containing other transmitters but did not cause these neurons to turn brown. Another possibility not excluded by our results is that 5,7-DHT might have interfered with release of some transmitter critical for restraint-induced inhibition, which is colocalized with 5-HT in crayfish neurons (see, e.g., Siwicki and Kravitz, 1983). At present, however, no other transmitter is known to mimic 5-HT's inhibitory effects on the LG circuitry.

\section{Potential role of the neurotoxin's side effects}

Another, related, problem for our interpretation of the results is that the observed behavioral deficit might have been due to some side effect of the 5,7-DHT injections. Unquestionably, the neurotoxin had side effects. Thus, the abdominal musculature of the neurotoxin-treated crayfish often turned yellowish (in normal animals its color is clear or whitish), and in some animals the carapace was pulled slightly apart from the abdominal exoskeleton. Furthermore, the 5,7-DHT-treated animals occasionally appeared sluggish and some eventually died. However, we have shown that 5,7-DHT does not lower the firing threshold of the LGs in unrestrained crayfish (see Fig. 8). This result suggests that the neurotoxin's effect on the threshold of the LGs in restrained crayfish was due to disruption of the system producing inhibition of the LGs, and not to drug-induced sickness, since the 5,7-DHT-treated animals in the restrained and unrestrained groups were, presumably, equally sick. Nevertheless, the possibility that the circuitry mediating restraint-induced inhibition might be sensitive (more sensitive than the basic pathway mediating escape itself) to some nonspecific effect of the neurotoxin cannot be entirely discounted.

\section{Modulatory role of central 5-HT in the crayfish's behavior}

Recent data from work on lobsters and crayfish have been taken to indicate that the monoamines octopamine and 5-HT may play a command role in the postural behavior of these crustaceans (Harris-Warrick and Kravitz, 1984; Livingstone et al., 1980). Specifically, central octopamine and 5-HT are thought to activate the motor circuitry for generating tonic extension and flexion. The present data, together with those of Glanzman and Krasne (1983), suggest a different type of behavioral role for these monoamines: Rather than subserving command or activational roles, they appear to subserve modulatory roles. Thus, neither octopamine nor 5-HT, when applied to the crayfish's CNS, drives the LG escape system. Moreover, after lesioning of central serotonergic neurons, the excitability of the LGs is altered only under circumstances (i.e., restraint) that normally produce inhibitory modulation of the escape system. In other words, the apparent effect of depleting the 5-HT within the crayfish's CNS is to strip away a specific modulatory overlay to the escape reaction while leaving the basic reaction itself intact.

It will be interesting to apply the technique of 5,7-DHT lesioning to discover how widespread are the inhibitory effects of the serotonergic modulatory system. Although we only studied the effects of 5,7-DHT injections on the LG response, restraint also suppresses other categories of crayfish escape, particularly those mediated by the medial giant fibers and by the so-called "non-giant" system (Krasne and Wine, 1975). Moreover, 5-HT might mediate other types of behavioral inhibition in the crayfish. As was mentioned earlier, the LG response is inhibited not only when the crayfish is restrained, but also when the animal is feeding (Krasne, 1983). Injections of 5,7-DHT might therefore also interfere with this feeding-induced inhibition of escape.

Researchers studying the modulation of invertebrate behaviors have proposed a variety of facilitatory roles for 5-HT. Thus, 5-HT is thought to mediate sensitization of Aplysia's gill withdrawal (Kandel et al., 1981) and the behavioral arousal associated with feeding in Aplysia (Kupfermann and Weiss, 1981) and the leech (Lent and Dickinson, 1984b). In contrast, researchers studying modulation of vertebrate behaviors have commonly proposed inhibitory roles for 5-HT (e.g., Davis and Sheard, 1974; Luine et al., 1983; McIntosh and Barfield, 1984; Zemlan et al., 1973; but see Davis et al., 1980). Our data suggest a role for 5-HT that is analogous to its proposed role in many vertebrate behaviors, i.e., that of a behavioral inhibitor. Of course, 5-HT might subserve both excitatory and inhibitory modulatory roles in the crayfish's behavior. For example, while 5-HT may inhibit escape, it may also facilitate other behaviors such as feeding.

\section{7-DHT injections: A technique for localizing} serotonergic cells in vivo

Obviously, it would be desirable to be able to study the 5-HTcontaining neurons of the crayfish's nerve cord electrophysiologically. However, localization of these neurons in living tissue from maps based on immunohistochemical results is problematic. Pretreatment with 5,7-DHT offers a simple alternative technique for localizing 5-HT-containing cells in vivo. Neurotoxin-treated serotonergic cells in the crayfish turn brown without any histochemical processing. Since the physiological inputs and membrane properties of comparably produced brown serotonergic neurons in adult leeches appear normal (Lent and Dickinson, 1984a), it may prove possible to use toxin-treated animals to develop electrophysiological criteria for identifying 5-HTcontaining cells in the crayfish's CNS.

Note added in proof. R. Fricke and J. McDonald (personal communication) have recently found a cluster of small 5-HT. immunoreactive cell bodies in the sixth abdominal ganglion using whole-mount preparations of juvenile crayfish. The distinctive appearance of this cluster of immunoreactive cells, as well as its position (at the base of the sixth ganglion near the seventh root's exit point), leaves little room for doubt that this 
is the same group of cells that turns brown following 5,7-DHT treatment (Fig. 4C). This finding reinforces the notion that 5,7DHT selectively lesions 5-HT-containing neurons in crayfish. Together with our inability to see immunoreactive staining of cells in the sixth abdominal ganglion, it also suggests that the neurotoxin's access to elements of the serotonergic system within the central ganglia of adult crayfish is superior to that of the 5-HT antibody, at least when the antibody is used in standard whole-mount immunohistochemical preparations.

\section{References}

Baumgarten, H. G., S. Jenner, A. Björklund, H. P. Klemm, and H. G. Schlossberger (1982) Serotonin neurotoxins. In Biology of Serotonergic Transmission, N. N. Osborne, ed., pp. 249-277, Wiley, New York.

Beltz, B. S., and E. A. Kravitz (1983) Mapping of serotonin-like immunorcactivity in the lobster nervous system. J. Neurosci. 3: 585602.

Beltz, B., J. S. Eisen, R. Flamm, R. M. Harris-Warrick, and E. Marder (1984) Serotonergic innervation and modulation of the stomatogastric ganglion of three decapod crustaceans (Panulirus interruptus, Homarus americanus and Cancer irroratus). J. Exp. Biol. 109: 3554.

Breen, C. A., and H. L. Atwood (1983) Octopamine-a neurohormone with presynaptic activity-dependent effects at crayfish neuromuscular junctions. Nature 303: 716-718.

Davis, M., and M. H. Sheard (1974) Habituation and sensitization of the rat startle response: Effects of raphe lesions. Physiol. Behav. 12. 425-431.

Davis, M., D. I. Astrachan, and E. Kass (1980) Excitatory and inhibitory cffects of serotonin on sensorimotor reactivity measurcd with acoustic startle. Science 209: 521-523.

Elofsson, R. (1983) 5-HT-like immunoreactivity in the central nervous system of the crayfish, Pacifastacus leniusculus. Cell Tissue Res. 232: 221-236.

Elofsson, R., L. Laxmyr, E. Roengren, and C. Hansson (1982) Identification and quantitative measurements of biogenic amines and DOPA in the central nervous system and hemolymph of the crayfish Pacifastacus leniusculus (Crustacea). Comp. Biochem. Physiol. $71 \mathrm{C}$ : 195-201.

Fischer, L., and E. Florey (1983) Modulation of synaptic transmission and excitation-contraction coupling in the opener muscle of the crayfish, Astacus leptodactylus, by 5 -hydroxytryptamine and octopamine. J. Exp. Biol. 102: 187-198.

Glanzman, D. L., and F. B. Krasne (1983) Serotonin and octopamine have opposite modulatory effects on the crayfish's lateral giant escape reaction. J. Neurosci. 3: 2263-2269.

Glover, J. C., and A. P. Kramer (1982) Serotonin analogue selectively ablates identified neurons in the leech embryo. Science $216: 1012-$ 1014.

Gunther, J., D. L. Glanzman, and F. B. Krasne (1984) Evidence that serotonin mediates restraint-induced inhibition of the crayfish's lateral giant escape response. Soc. Neurosci. Abstr. 10:626.

Harris-Warrick, R. M., and E. A. Kravitz (1984) Cellular mechanisms for modulation of posture by octopamine and serotonin in the lobster. J. Neurosci. 4: 1976-1993.

Jahr, C. E., and R. A. Nicoll (1982) Noradrenergic modulation of dendrodendritic inhibition in the olfactory bulb. Nature 297: $227-$ 229.

Kandel, E. R., M. Klein, C. H. Bailey, R. D. Hawkins, V. F. Castellucci, B. W. Lubit, and J. H. Schwartz (1981) Serotonin, cyclic AMP, and the modulation of the calcium current during behavioral arousal. In Serotonin Neurotransmission and Behavior, B. L. Jacobs and A. Gelperin, eds., pp. 211-254, MIT, Cambridge, MA.

Krasne, F. B. (1983) Excitability of the crayfish lateral giant escape circuit is depressed during feeding. Soc. Neurosci. Abstr. 9: 754.

Krasne, F. B., and D. L. Glanzman (in press) Sensitization of the crayfish lateral giant escape reaction. J. Neurosci.

Krasne, F. B., and J. J. Wine (1975) Extrinsic modulation of crayfish escape behavior. J. Exp. Biol. 63: 433-450.

Kravitz, E. A., S. Glusman, M. S. Livingstone, and R. M. Harris-War- rick (1981) Serotonin and octopamine in the lobster nervous system: Mechanism of action at neuromuscular junctions and preliminary behavioral studies. In Serotonin Neurotransmission and Behavior, B. L. Jacobs and A. Gelperin, eds., pp. 189-210, MIT, Cambridge, MA. Kristan, W. B., Jr., and M. P. Nusbaum (1982/1983) The dual role of serotonin in leech swimming. J. Physiol. (Paris) 78: 743-747.

Kupfermann, I., and K. R. Weiss (1981) The role of serotonin in arousal of feeding behavior of Aplysia. In Serotonin Neurotransmission and Behavior, B. L. Jacobs and A. Gelperin, eds., pp. 255-287, MIT, Cambridge, MA.

Lent, C. M., and M. H. Dickinson (1984a) Retzius cells retain functional membrane properties following "ablation" by the neurotoxin 5,7-DHT. Brain Res. 300: 167-171.

Lent, C. M., and M. H. Dickinson (1984b) Serotonin integrates the feeding behavior of the medicinal leech. J. Comp. Physiol. A 154 : 457-471.

Libet, B. (1979) Dopaminergic synaptic processes in the superior cervical ganglion: Models for synaptic actions. In The Neurohiology of Dopamine, A. S. Horn, J. Korf, and B. H. C. Westerink, eds., pp. 453-474, Academic, New York.

Livingstone, M. S., R. M. Harris-Warrick, and E. A. Kravitz (1980) Serotonin and octopamine produce opposite postures in lobsters. Science 208: 76-79.

Livingstone, M. S., S. F. Schaeffer, and E. A. Kravitz (1981) Biochemistry and ultrastructure of serotonergic nerve endings in the lobster: Serotonin and octopamine are contained in different nerve endings. J. Neurobiol. 12: 27-54.

Luine, V. N., M. Frankfurt, T. C. Rainbow, A. Biegon, and E. Azmitia (1983) Intrahypothalamic 5,7-dihydroxytryptamine facilitates feminine sexual behavior and decreases $\left[{ }^{3} \mathrm{H}\right]$ imipramine binding and 5HT uptake. Brain Res. 264: 344-348.

Madison, D. V., and R. A. Nicoll (1982) Noradrenaline blocks accommodation of pyramidal cell discharge in the hippocampus. Nature 299: 636-638.

McIntosh, T. K., and R. J. Barfield (1984) Brain monoaminergic control of male reproductive behavior. I. Serotonin and the post-ejaculatory refractory period. Behav. Brain Res. 12: 255-265.

Nässel, D. R., and K. Elekes (1985) Serotonergic terminals in the neural sheath of the blowfly nervous system: Electron microscopical immunocytochemistry and 5,7-dihydroxytryptamine labelling. Neuroscience 15: 293-307.

O'Shea, M., and P. D. Evans (1979) Potentiation of neuromuscular transmission by an octopaminergic neuron in the locust. J. Exp. Biol. 79: 169-190.

Paupardin-Tritsch, D., P. Deterre, and H. M. Gerschenfeld (1981) Relationship between two voltage-dependent serotonin responses of molluscan neurons. Brain Res. 217: 201-206.

Roberts, A. (1968) Recurrent inhibition in the giant-fiber system of the crayfish and its effect on the excitability of the escape response. J. Exp. Biol. 48: 545-567.

Rosen, S. C., I. Kupfermann, R. S. Goldstein, and K. R. Weiss (1983) Lesion of a serotonergic modulatory neuron in Aplysia produces a specific defect in feeding behavior. Brain Res. 260: 151-155.

Siwicki, K. K., and E. A. Kravitz (1983) Proctolin in lobsters: General distribution and co-localization with serotonin. Soc. Neurosci. Abstr. 9: 313.

Van Harreveld, A. (1936) A physiological solution for fresh water crustaceans. Proc. Soc. Exp. Biol. Med. 34: 428-432.

Watanabe, S., and K. Koketsu (1973) 5-HT hyperpolarization of bullfrog sympathetic ganglion cell membrane. Experientia 29: 1370-1372.

Willard, A. L. (1981) Effects of serotonin on the generation of the motor pattern for swimming by the medicinal leech. J. Neurosci. 1 : 936-944.

Wine, J. J., and F. B. Krasne (1972) The organization of escape behavior in the crayfish. J. Exp. Biol. 56: 1-18.

Wine, J. J., and F. B. Krasne (1982) The cellular organization of crayfish escape behavior. In The Biology of Crustacea, Vol. 4, D. C. Sandeman and H. L. Atwood, eds., pp. 241-292, Academic, New York.

Zemlan, F. P., I. L. Ward, W. R. Crowley, and D. L. Margules (1973) Activation of lordotic responding in female rats by suppression of serotonergic activity. Science 179: 1010-1011. 